\title{
Modelling of Residential Heating Systems using a Phase Change Material Storage System
}

\author{
Corinna Leonhardt Dirk Müller \\ Institute for Energy Efficient Buildings and Indoor Climate, \\ E.ON Energy Research Center, RWTH Aachen University \\ Jaegerstr. 17/19, 52066 Aachen, Germany \\ cleonhardt@eonerc.rwth-aachen.de
}

\begin{abstract}
Modern heating systems for buildings need a supply temperature of approximately $35{ }^{\circ} \mathrm{C}$. Standard heat storage systems do not work very efficiently with small supply temperature differences, because of the low sensible heat storage capacity. In contrast to the sensible heat storage a phase change material (PCM) storage system uses the phase change process to store energy at small temperature differences [3], [4], [5]. In this paper a thermo hydraulic model of a PCM storage is developed and implemented by using Modelica, so that dynamic modelling is possible. To show the advantages of latent heat storage (LHS) the PCM storage model has been combined to build a standard heat pump system model with a PCM storage instead of a sensible water storage and the overall system is analysed.
\end{abstract}

Keywords: PCM; latent heat storage; heat pump; thermo hydraulic modelling

\section{Introduction}

The topic of this research project is to study a latent heat storage device for modern heating systems with a supply temperature of approximately $35^{\circ} \mathrm{C}$. The integration of the storage system into a heat pump system should equalize the work load profile leading to a higher yearly averaged coefficient of performance. Additionally, the necessary heat pump peak power will be decreased because the heat pump is able to load the storage system over night time. The center of research is the development of computer simulations to examine the feasibility and advantages of a latent heat storage system and of course to find an efficient way of using the power of the PCM.
The institute for Energy Efficient Buildings and Indoor Climate focuses on the research of the reduction of energy consumption of buildings and indoor climate. There are two main research groups in the institute. The first one works on energy systems and the second one focuses on room airflows and indoor comfort. According to these topics, one research approach for example is the thermo hydraulic modelling and simulation of energy systems and single components with Modelica. The institute uses and develops its own libraries [1]. Up to now one library for the thermal building behaviour and a second one for HVAC systems have been created. With the help of these libraries and the Modelica Standard Library a model of a phase change material storage system is created and analysed.

Heat Pump Latent Heat Storage Device Heating System

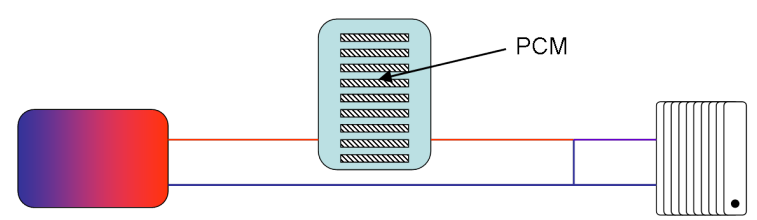

Figure 1: Heat Pump System in Combination with Latent Heat Storage

Heat Pump Sensible Heat Storage Device Heating System

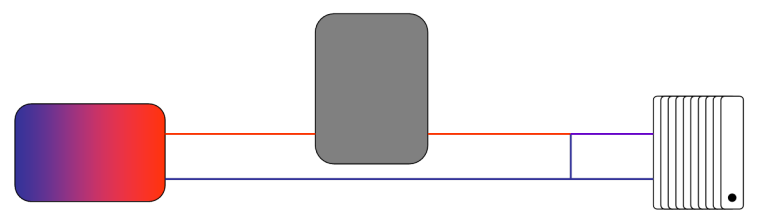

Figure 2: Heat Pump System in Combination with Sensible Heat Storage 
This paper describes the modelling and simulation of a latent heat storage device and its integration in a heat pump system. First the development of a PCM storage device is shown and then the single components of the heat pump systems are mentioned. Finally a comparison between a heat pump system with a sensible water storage to a heat pump system with a latent heat storage is made and the results are shown in this paper.

The pictures (fig. 1 and fig. 2) show a schematic view of the overall systems. The first system describes a simple residential heating system in combination with a latent heat storage (fig. 1). The second one is the reference case. Therefore, the whole set-up is equal to the first one, but this time the latent heat storage device is replaced by a simple buffer storage with thermal layering.

\section{Latent Heat Storage Device}

\subsection{Heat Transfer Equations}

A thermo hydraulic model of a latent heat storage device is developed. The model is based on energy balance, which is given by:

$$
\sum \dot{Q}_{i}=m_{\text {cell }} \cdot c_{\text {cell }} \cdot \frac{d T_{\text {cell }}}{d t}
$$

The sum of all heat fluxes $\dot{Q}_{i}$ is equal to the product of mass $m_{\text {cell }}$, heat capacity $c_{\text {cell }}$ and the derivative with respect to time of the temperature $\frac{d T_{\text {cell }}}{d t}$. In this paper a modified heat capacity is used and described by an arc tangent function [2] of the specific enthalpy:

$$
h=h_{t} \cdot\left[\frac{\arctan \left(\left(T-T_{t}\right) \cdot r_{t}\right)}{\pi}+0.5\right]+c \cdot\left(T-T_{0}\right)
$$

$$
\begin{array}{ll}
\multicolumn{2}{c}{\text { first term (latent heat part) }} \\
h_{t} & \text { specific enthalpy of transition } \\
T_{t} & \text { temperature of transition } \\
r_{t} & \text { width of transition }
\end{array}
$$

\section{second term (sensible heat part)}

$c$ specific heat capacity

$T_{0} \quad$ reference temperature of the system

So that the heat capacity is given by:

$$
c_{p}=\frac{h_{\text {trans }}}{\pi \cdot\left[\left(\left(T-T_{\text {trans }}\right) \cdot r_{\text {trans }}\right)^{2}+1\right]}+\widehat{c_{p}}
$$

Fig. 3 shows an example of the heat capacity and enthalpy function of an invented PCM, which is based on a typical paraffin with a phase change by $320 \mathrm{~K}$. This latent heat storage material has a characteristic phase change temperature of $320 \mathrm{~K}$, a phase change enthalpy of $180 \mathrm{~kJ} / \mathrm{kg}$ and a heat capacity of $2.4 \mathrm{~kJ} /(\mathrm{kgK})$. For the width of the peak in the function of the specific heat capacity curve the factor $r_{t}$ is set to the value two.

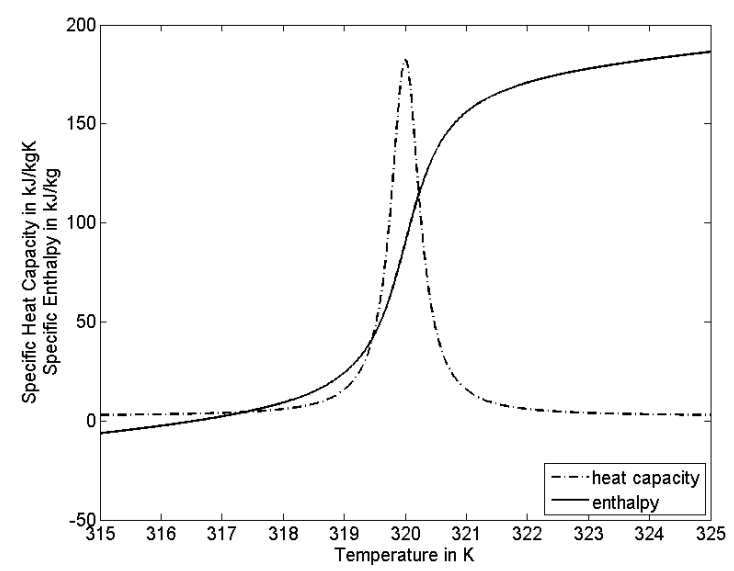

Figure 3: Specific heat capacity and enthalpy

\subsection{Model of Latent Heat Storage}

With the help of these functions a new latent heat storage model has been created. The new latent heat storage device consists of a box with several PCM plates, which are flowed by water. The new library consists of several sub packages, for example: components, database and systems.

The components package includes all parts of a PCM storage. For instance it includes the plates of different phase change materials and several latent heat storage devices, which differ in the set-up and in the number of PCM plates. Generally a PCM storage device is made up of several single plates in combination with standard pipes of the Modelica Fluid Library (fig. 4). The figure shows one example of latent heat storage device, which exists of 12 plates of PCM and a water flux, which flows in a meandering course across the plates.

The plates are filled with the phase change material. 


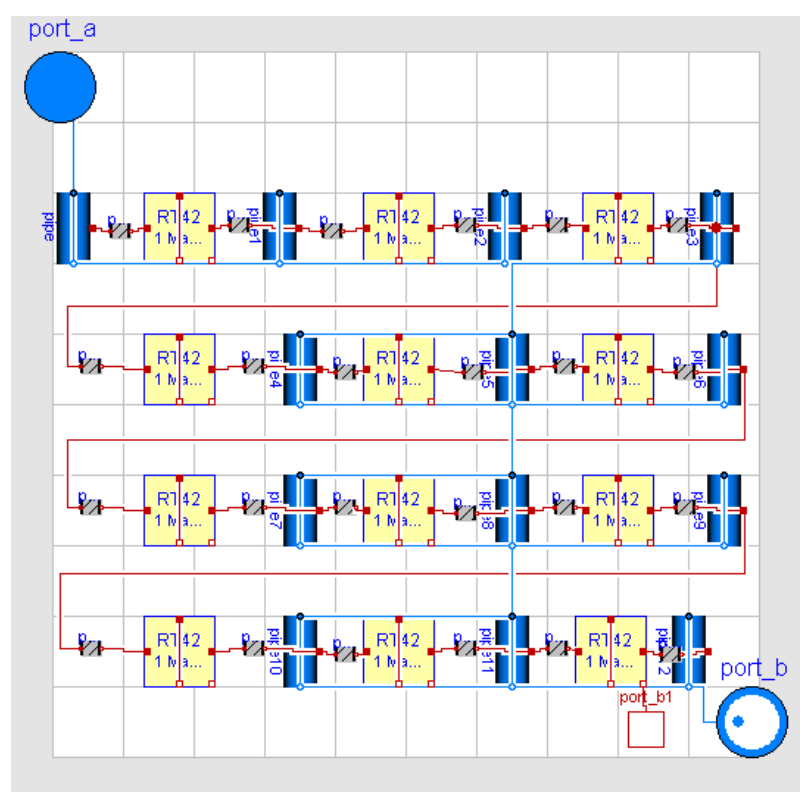

Figure 4: Structure of a PCM Storage Device

Up to now there is no conduction and no mass of the plate itself implemented, so that heat transfer from the latent heat storage material to the pipe wall is ideal. In order to get the best layout of the PCM storage plate the design of an existing storage plate for cooling systems is adapted to the heating system requirements.

One latent heat storage plate can be divided in two directions (see fig. 5).
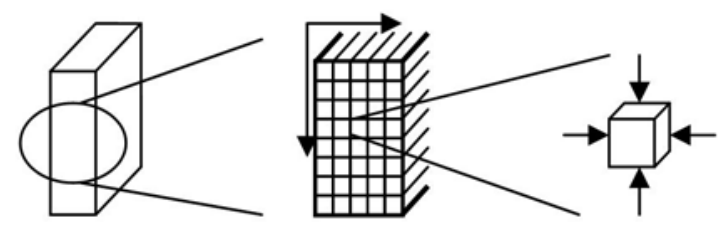

Figure 5: Structure of the Plates

In order to get the opportunity to describe the behaviour of one latent heat storage plate in two directions, it consists of discrete elements (finite volumes). Every single element is described by one energy balance (see fig. 6), which is realized by one capacity block and four conductivity blocks, so that the temperature distribution in the latent heat storage device can be examined.

Fig. 6 shows the four heat conduction elements and one capacity block, which are used. The single

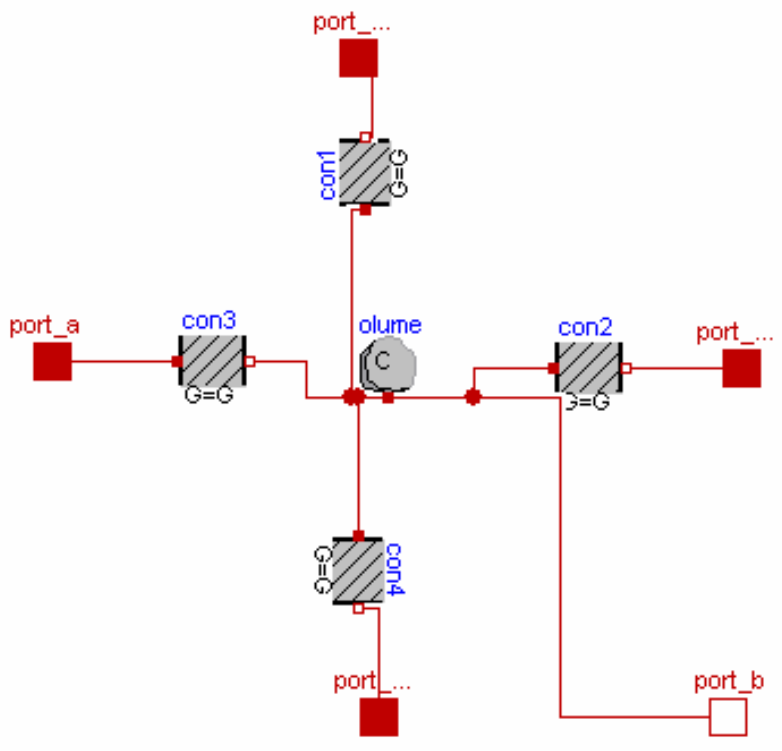

Figure 6: Structure of the PCM-Volume

volumes are connected with each other, so that the whole plate is created.

Due to the fact that thermodynamic properties change during the phase change process the capacity block and the conduction block of the Modelica Standard Library are adapted (see Model PCM _ capacity and Model PCM _ conductivity).

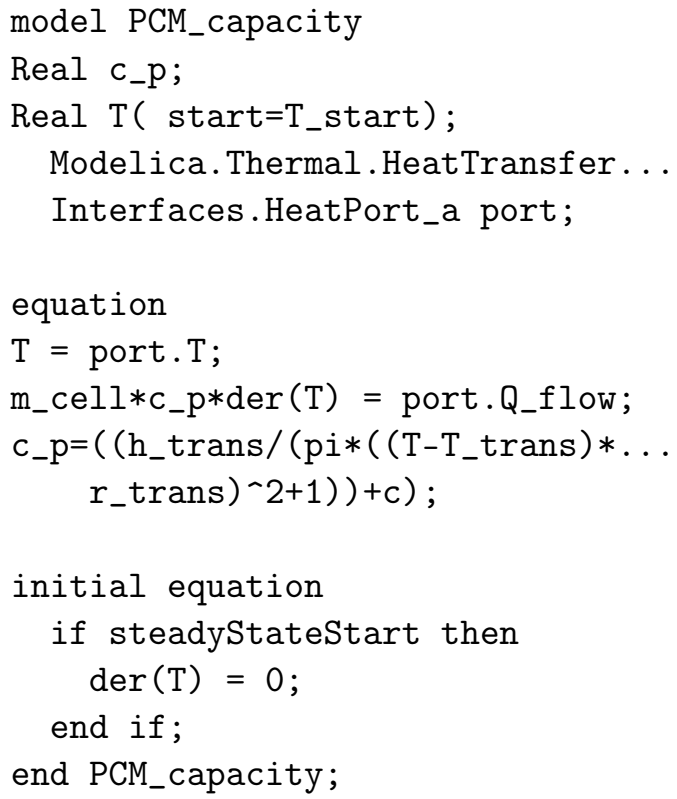

The capacity block is duplicated and modified by using the equation (3) mentioned before (see Model PCM _ capacity, equation section). 
Another point is that the conductivity of the latent heat storage material is a function of the actual state of aggregation, so that the heat conduction, lambda, is a function of the amount of the liquid and solid mass fraction as shown in the model PCM _ conductivity.

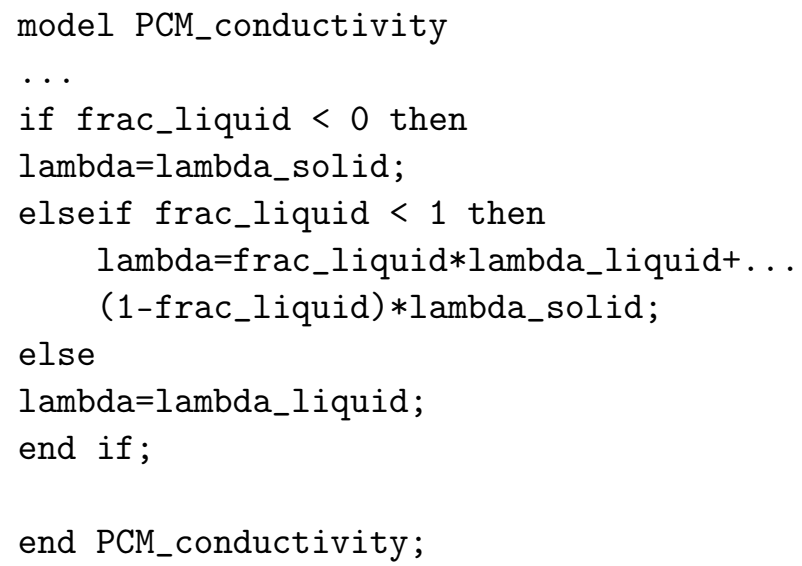

Fig. 7 describes another aspect, which has to be kept in mind by using a latent heat storage device, the question of the amount of PCM, which has to be used to keep the building warm.

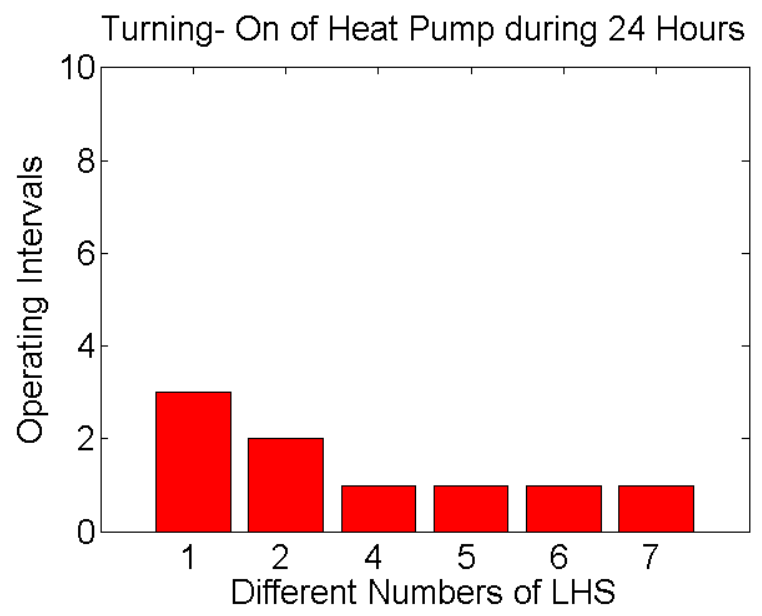

Figure 7: Comparison of different numbers of LHS

The simulation set-up is based on a cool day and a building, which is built up of five identical rooms. As the name already suggests, the simple house model describes the behaviour of a residential building. It consists of five rooms with the same geometrical and thermo hydraulic qualities.
As fig. 7 shows, the heat pump has to turn on three times to keep the rooms warm, if there is only one latent heat storage device. According to this, the heat pump has to turn on once, if there are 7 PCM storage devices integrated.

For the actual study six latent heat storage devices have been taken, which means a total amount of mass of PCM of about $170 \mathrm{~kg}$.

\section{Heat Pump System}

At the Institute for Energy Efficient Buildings and Indoor Climate an independent library for the whole heat pump system has been developed [1]. This enables us to take off all components for the heat pump system, there. Therefore all components can be directly put together to an overall system (see fig. 8 and fig. 9).

The heat pump model is described in detail in paper [1]. This model consists of two heat exchangers (condenser and evaporator). Both heat exchangers allow changing energy between the heat pump cycle and the medium of the heating cycle.

The standard buffer storage is also a component, which can be taken off the heat pump library. It consists of several layers. Within the model any number of them can be chosen. In this study the buffer storage consists of five layers, so that thermal layering is also taken into account.

The heating cycle is controlled by a heating curve, which is calculated in respect to the ambient temperature.

\section{Combined Simulation}

A comparison of two heat pump systems has been made. The first system consists of simple buffer storage with thermal layering and in contrast to the first system, in the second one the buffer storage is replaced by a latent heat storage device.

In fig. 8 the buffer storage is presented in the middle. On the left the heat pump cycle is presented and on the right the heating cycle can be seen. 


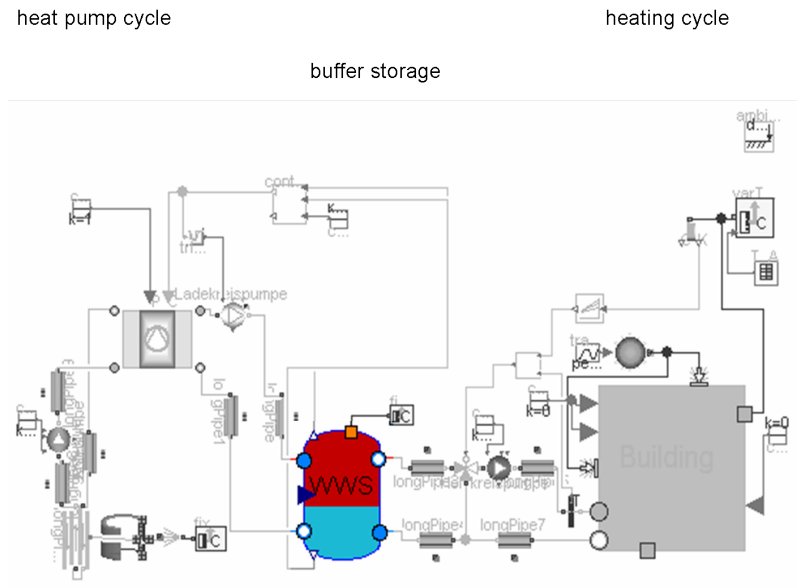

Figure 8: Heat pump system with buffer storage

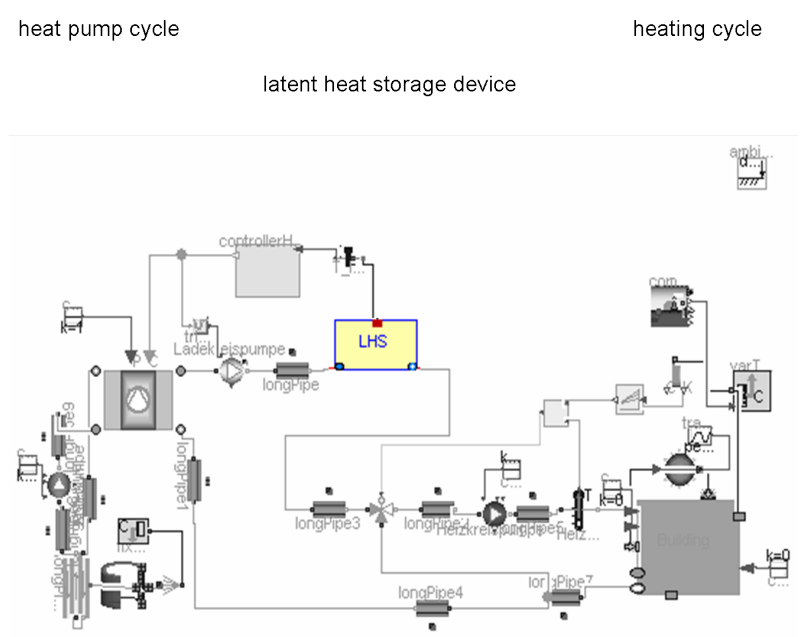

Figure 9: Heat pump system with latent heat storage

The set-up of fig. 9 is built up analogously. Only the storage system, the buffer storage, is replaced by a latent heat storage device.

Both systems are simulated for a cool day in May and the results are shown in fig. 10 and fig. 11.

\subsection{Results}

Fig. 10 shows the results of the first simulation run of the two systems (fig. 8 (warm water storage) (WWS)) and fig. 9 (latent heat storage) (LHS)) and in fig. 11 the results after an optimization of the overall system are described. In the first diagram of fig. 10 and fig. 11 the results of the heat pump power are described. The second diagram shows the heat pump condenser temperature and the last diagram shows the ambient temperature and the room temperature.
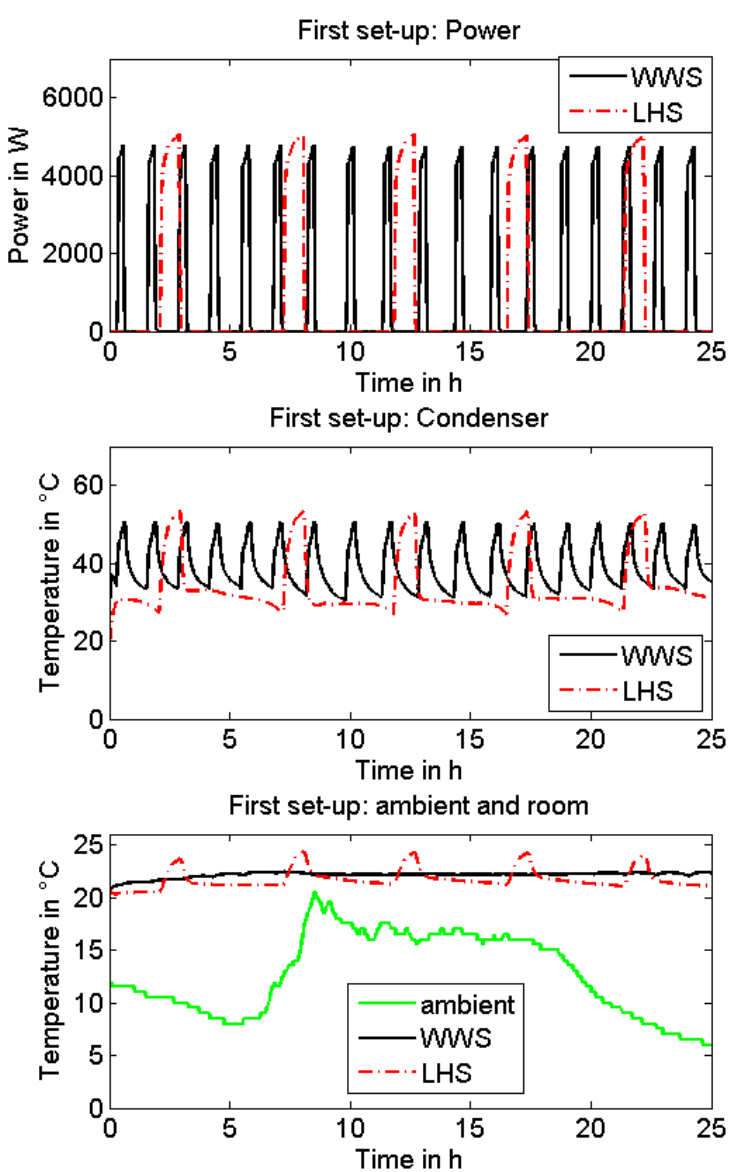

Figure 10: Results of first set-up

The results of fig. 10 and fig. 11 show that the latent heat storage device improves the heat pump behaviour. On the one hand the switching between on and off of the heat pump can be reduced by integrating a PCM storage instead of the buffer storage, so that heating costs can be spared. On the other hand it is even possible to reduce the amount of energy, because of the low supply temperature, which reduces the energy losses. So another effect should be lower heat pump temperatures, but up to now they are only a little bit lower after the optimization, but this still has to be analysed.

\subsection{Conclusions}

The first simulations of the latent heat storage device show the potential of such a storage system and that its integration into heat pump systems can decrease the heat pump power. 

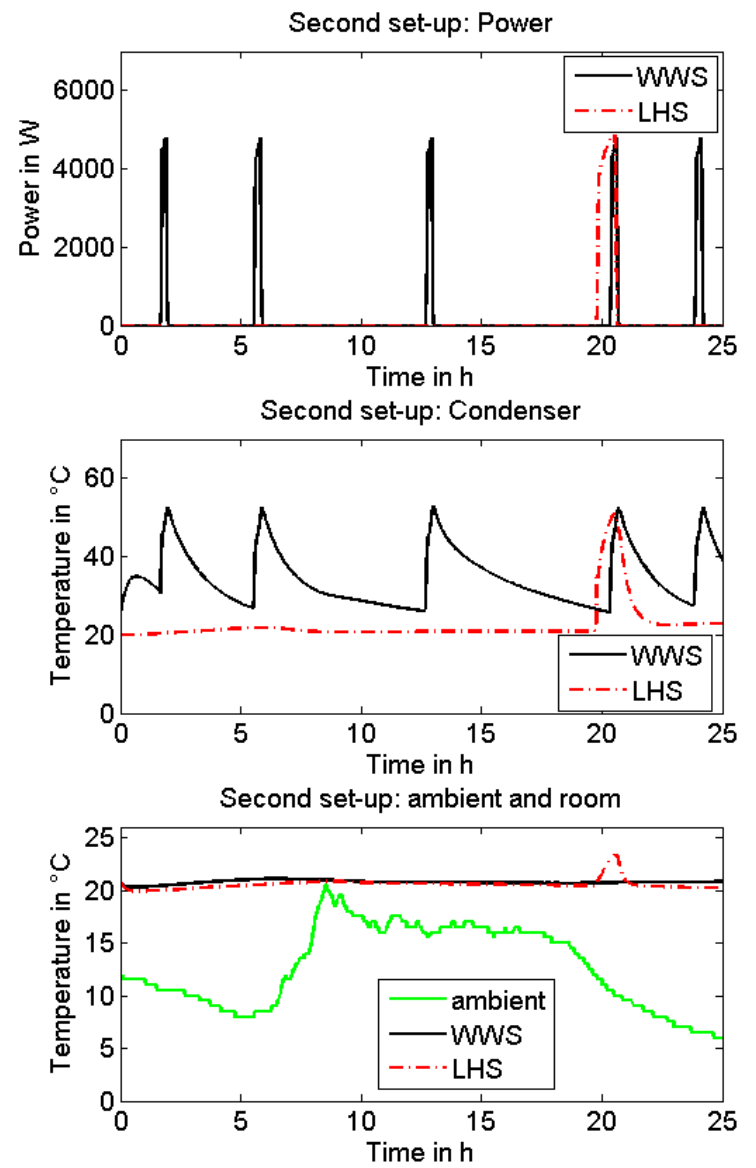

Figure 11: Results of the second set-up

The existing latent heat storage component itself will be improved, for example by adding the conduction and mass of the storage plate. Another point is that the heat transfer to the water in respect to the specific surface area of the plates will be integrated.

One further application, which will be simulated, is the combination with a solar thermal collector. And another interesting addition of the existing simulation model would be the integration of a domestic hot water tank to the overall system.

\section{Acknowledgement}

The authors would like to thank the E.ON gGmbH for the financial support of the project.

\section{References}

[1] Huchtemann, K. Advanced simulation methods for heat pump systems: Modelica 2009, September 20-22.

[2] Buschle J. Analysis of steam storage systems using Modelica: Modelica 2006, September 4-5.

[3] Fachinformationszentrum Karlsruhe (Hg.) Wärmespeicher: BINE- Informationsdienst, Karlsruhe 2005

[4] Mehling, Cabeza Heat and Cold Storage with PCM: Heat and Mass Transfer, Springer, Berlin 2008

[5] htttp://www.zae-bayern.de / deutsch / abteilung$1 /$ arbeitsgebiete / latentwaermespeicher / einführungg.html (Februar 2009) 